\title{
Heterogeneous Modulation for Trading-off Energy Balancing with Bandwidth Efficiency in Hierarchical Sensor Networks
}

\author{
Maryam Soltan, Inkwon Hwang, and Massoud Pedram \\ University of Southern California \\ \{soltan, inkwonhw, pedram\}@usc.edu
}

\begin{abstract}
With power efficiency and lifetime awareness becoming critical design concerns in wireless sensor networks (WSN), it has become essential to exploit the physical layer characteristics at the system deployment stage. This paper focuses on how certain physical layer attributes can affect both the lifetime and the end-to-end delay in a hierarchical WSN. We present a heterogeneous modulation scheme and report its impact on the spatial distribution of energy dissipation and the resulting network lifetime. Moreover, we study how this heterogeneous modulation scheme affects the end-to-end delay due to inherent trade-offs in power efficiency and bandwidth efficiency of the different modulation schemes.
\end{abstract}

\section{Introduction and Related work}

Wireless Sensor Networks (WSN) are used for different applications where a large number of battery operated sensors with some communication capability collect data and send it towards a base station. Early failure of a number of sensors in a particular region can lead to degradation in the quality of service. A design challenge is to come up with an optimized communication infrastructure as well as signaling schemes and radio specifications to efficiently use each sensor's energy resource, and thereby, extend the network lifetime under end to end delay constraints.

Lots of research has focused on energy-aware design to better utilize the limited communication and computation capabilities of the sensors [1-10]. There has been a growing interest in hierarchical WSN architectures to reduce the communication burden on the sensors and hence increase the network lifetime [25]. Although significant research has focused on energy efficient design in different levels of network protocol stack, much less work has been done on the effects of physical layer characteristics in the deployment architectures and overall system design. Modulation scaling and adaptive modulation techniques are introduced in [8-10] where the modulation level is adjusted to reduce the energy per bit. The target bit error rate is increased in order to reduce the required energy per bit based on either data traffic [8] or bit rate requirements [10]. The impact of using modulation scaling on data delivery latency and data loss is considered in [9]. In modulation scaling techniques, one common modulation scheme is considered for all the nodes and the level is adjusted to achieve lower required energy per bit, as long as the application can accommodate increased bit error rate. However, we consider a location-aware heterogeneous modulation selection among different sensors. We study the implications of the inherent trade-offs in power efficiency versus bandwidth efficiency for different modulation schemes and show how a heterogeneous modulation scheme can affect the data delivery delay and the overall spatial distribution of energy consumption. This technique can hence be used in system design and deployment phase to increase the network lifetime.

In Section 2, background material and our motivation are presented. System description and approach are described in Section 3 followed by simulation results in Section 4. Finally, concluding remarks are presented in Section 5.

\section{Background and Motivation}

We review the concept of bandwidth efficiency and the trade-off between power and bandwidth efficiency for different modulation schemes [11][12] as a motivation for our heterogeneous modulation scheme.

\subsection{Bandwidth efficiency}

Bandwidth efficiency is a key characteristic of any modulation scheme and is defined as the number of bits per second that can be transmitted over $1 \mathrm{~Hz}$ of system bandwidth. Modulation schemes can broadly be divided into multiphase and orthogonal schemes. For multiphase modulations with ideal rectangular baseband pulse shaping, the minimum bandwidth 
required to transmit one symbol every $T$ seconds is $B_{\min }=1 / T(\mathrm{~Hz})$. With an M-ary multiphase modulation, each symbol will contain $k=\log _{2} M$ bits, and hence the bit rate can be obtained as $R_{b}=k / T=k B_{\min }$. Therefore, the maximum bandwidth efficiency of M-ary multiphase modulations is given by:

$$
R_{b} / B_{\min }=\log _{2} M \quad(\mathrm{bps} / \mathrm{Hz})
$$

On the other hand, with an M-ary orthogonal modulation, $M=2^{k}$ orthogonal carriers with minimum frequency separation of $1 / 2 T$ are required to transmit $k$ bits over $T$ seconds. Therefore the total minimum required bandwidth is given by:

$$
B_{\min }=\frac{M}{2 T}=\frac{M}{2\left(k / R_{b}\right)}=\frac{M}{2 \log _{2} M} R_{b}
$$

So the maximum bandwidth efficiency is:

$$
R_{b} / B_{\text {min }}=\left(2 \log _{2} M\right) / M \quad \text { (bps/Hz) }
$$

Given the bit rate, Table 1 shows the theoretical limits on the minimum required bandwidth for some modulation schemes. In practice, presence of intersymbol interference mandates larger signaling bandwidths for a given bit rate, or equivalently forces a lower bit rate for a given available channel bandwidth.

\subsection{Trade-off between bandwidth efficiency and power efficiency}

As a modulation scheme becomes more power efficient, it requires larger bandwidth for a given data rate. This fundamental trade-off can be explained using Shannon's channel capacity theorem [13]. Shannon showed that the maximum bit rate that can reliably be transmitted over an additive white Gaussian noise (AWGN) channel with bandwidth $B$ is

$$
R_{b}<B \log _{2}\left(1+P / N_{0} B\right)
$$

where $R_{b}$ is bit rate in bits per second, $P$ is power in Jules per sec, and $N_{0}$ is the noise power spectral density in Watts per Hz. Given energy per bit $E_{b}=P / R_{b}$ :

$$
\frac{R_{b}}{B}<\log _{2}\left(1+\frac{E_{b}}{N_{0}} \frac{R_{b}}{B}\right) \Rightarrow \frac{E_{b}}{N_{0}}>\frac{2^{R_{b} / B}-1}{R_{b} / B}
$$

Figure 1 shows this fundamental limit curve highlighting the fact that larger $E_{b} / N_{0}$ is required for higher bandwidth efficiency. A minimum target $E_{b} / N_{0}$ along with the bandwidth efficiency of a specific modulation scheme corresponds to a point somewhere below the limit curve. A physical layer design challenge is to come up with modulation and coding schemes that can move closer to this limit curve.

\subsection{Motivation}

We exploit the trade-off between bandwidth and power efficiency and study how a location-aware heterogeneous modulation scheme can be used in order to trade energy balance for bandwidth efficiency and
Table 1: Bandwidth Efficiency of Modulations

\begin{tabular}{|c|c|}
\hline Modulation Method & $\begin{array}{c}\text { Min. Required Channel } \\
\text { Bandwidth (Hz) }\end{array}$ \\
\hline BPSK, DBPSK, BFSK, 4-FSK & $1.0^{*}$ (bit rate in bps) \\
\hline QPSK, DQPSK, OQPSK & $0.5^{*}$ (bit rate in bps) \\
\hline
\end{tabular}

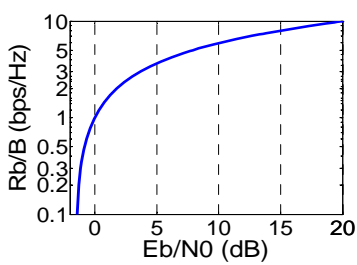

Figure 1: Bandwidth vs. Power Efficiency Limit Curve

hence the end-to-end delay in a hierarchical WSN.

\section{System Description and Approach}

We consider a hierarchical WSN consisting of event aggregator relays (EAR) and sensors [5]. The sensors are simple low power sensing devices with some communication capability. EARs are more powerful with higher communication capabilities. EARs form a network infrastructure to collect data from a cluster of sensors in their coverage and relay it to the base station. We consider a deterministic multiple-access approach based on a combined Frequency Division Multiple Access (FDMA) and Time Division Multiple Access (TDMA) scheme. Hence, a (frequency, timeslot) pair is assigned for each sensor-to-EAR communication channel, avoiding co-channel interference among sensors within a cluster. EAR-tobase station communications are done on different channels. The total frequency bandwidth available for each cluster is fixed and acts as a constraint, which, given the minimum required channel bandwidth, specifies the number of available frequency channels in each cluster.

In the following subsections, we discuss our approach and study how delay and lifetime can be affected by modulation schemes.

\subsection{Heterogeneous modulations and lifetime}

Lifetime of the network depends on the lifetime of each cluster. By definition, the lifetime of a cluster is finished when the percentage of live sensors falls below a pre-specified threshold, such that the cluster can no longer satisfy the required quality of sensing. To increase the lifetime of the network, we try to balance the energy distribution within each cluster.

Energy dissipation due to data transmission is a large percentage of the overall energy consumption. The required energy per transmitted bit in sensor $i$ is, 


$$
e_{T x}(i)=k_{T x} \cdot E_{b} \cdot\left(4 \pi d_{e(i), i} / \lambda_{W}\right)^{\beta_{e(i), i}}
$$

where $k_{T x}$ is a constant, which depends on antenna gain and the output amplifier efficiency of the sensor as well as the receiver Noise Figure of the EAR node. $E_{b}$ is the needed energy per bit at the receiver in order to satisfy the bit error rate (BER) requirement. $d_{e(i), i}$ and $\beta_{e(i), i}$ respectively are the distance and the path loss exponent between sensor $i$ and its assigned EAR $e(i)$.

For any modulation scheme, the bit error rate can be characterized as a function of $E_{b} / N_{0}$ [11]. For a given bit error rate, there can be a large difference between the required $E_{b} / N_{0}$ for various modulation schemes. By proper selection of modulation schemes for different sensors across a cluster, we can reduce the average energy consumption and even out the energy distribution over the coverage area. Each sensor may only support one modulation scheme while EARs are assumed to support multiple modulation schemes either via multiple receivers or via a single softwaredefined configurable receiver.

Considering $N_{s}$ sensors in a cluster with radius $R$ and EAR located in the center, we divide the sensors into two groups. For the sensors closer to the EAR within distance of $r$, we select a bandwidth efficient Modulation 1 with larger $E_{b} / N_{0}$. However, for farther away sensors, we select a less bandwidth efficient Modulation 2 that can achieve the same BER with smaller $E_{b} / N_{0}$. Therefore, if Modulation 1 requires $E_{b}$ Jules per bit for a given BER, Modulation 2 will achieve the same BER with $k E_{b}$ Jules per bit, where $k$ is smaller than 1. For example to achieve BER of 1e-4, required energy per bit for Binary phase Shift Keying (BPSK) is 0.55 of the energy per bit for Quadrature Phase Shift Keying (DQPSK) (i.e. $k=0.55$ )[12]. The average transmission energy per bit for the sensors located within distance $r$ with Modulation 1 is $e_{T x 2}=k_{T x} E_{b} \cdot\left(2 \pi r / \lambda_{W}\right)^{\beta}$ and for other sensors with Modulation 2 is $e_{T x 2}=k_{T x} \cdot k \cdot E_{b} \cdot\left(4 \pi(r+0.5(R-r)) / \lambda_{W}\right)^{\beta}$.

Although Modulation 2 is less bandwidth efficient, utilizing it for the more distant sensors, which have larger path loss and hence higher energy consumption, can result in better spatial balancing of the energy.

\subsection{Delay and time slot arrangements}

By definition, end-to-end delay is the delay between the time the sensor data is generated and the time it is delivered to the base station. In the case of single-hop communication for sensor-to-EAR and EAR-to-base station communication, the end-to-end delay is a function of channel access time. Given FDMA/TDMA channels, the channel access time for each node depends on the number of frequencies, duration and the number of available time slots in every frame (i.e.,

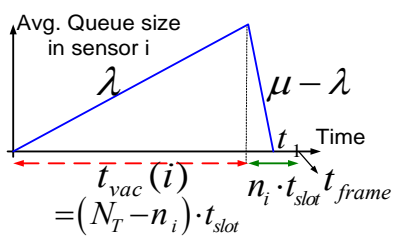

Figure 2: Temporal variation of queue size

TDMA channels for each frequency), and the number of time slots assigned to the node. These parameters need to be determined and optimized across the network in order to satisfy the sensing traffic load requirement, meet delay constraint for packet delivery, and honor channel bandwidth limitations.

To avoid co-channel interference, sensors sharing the same frequency channel need to communicate in different time slots. This will cause channel access delay. Every sensor collects the sensing information and stores it in a queue until it can start data transmission over the communication link. Hence, the communication link acts as a server with vacation [14] for the sensor. The higher the number of sensors sharing the same frequency, the longer the wait time will be for each sensor in accessing its channel.

Considering $N_{f}$ frequency channels over a total available bandwidth $B_{\text {cluster }}$ for each cluster, the bandwidth for each frequency channel is $B_{\text {cluster }} / N_{f}$. This bandwidth may include a guard band in order to avoid any adjacent channel interference in the cluster. Let's consider $N_{T}$ time slots with duration of $t_{\text {slot }}$ in every time frame of size $t_{\text {frame }}=t_{\text {slot }} \times N_{T}$. The total number of FDMA/TDMA channels available in the cluster is $N_{T} \times N_{f}$ and it should not be less than the number of sensors in that cluster to avoid co-channel interference. $N_{T}, N_{f}$, and $t_{\text {slot }}$ are the parameters that need to be optimized as part of the communication infrastructure design based on the application characteristics and specifically, the sensing rate and the expected channel access delay.

Let's consider a maximum of $N$ sensors sharing a frequency channel. For sensor $i$, let $\lambda_{i}$ and $\mu_{i}$ denote the average sensing rate and the data transmission bit rate, respectively. The maximum achievable transmission bit rate depends on the channel bandwidth and bandwidth efficiency of the selected modulation scheme (cf. Table 1). For sensor $i$, data is stored in the queue until the assigned time slot for that sensor arrive, and then data is transmitted towards the EAR. Assume that $n_{i}$ time slots are assigned to sensor $i$. Temporal variation of queue size for sensor $i$ during a time frame is shown in Figure 2. We model the queue for each sensor as $\mathrm{M} / \mathrm{D} / 1$ with vacation (see [14] for the queue models). In order to handle the sensing traffic and to avoid queue buildup, on average, data generated during 
Table 2: Simulation Results

\begin{tabular}{|c|c|c|c|c|}
\hline & & $\begin{array}{c}\text { Avg. } \\
\text { remaining } \\
\text { over init. } \\
\text { energy } \\
\text { after } 2638 \\
\text { days } \\
\end{array}$ & $\begin{array}{c}\text { Lifetime } \\
\text { (Days) } \\
30 \% \\
\text { dead } \\
\text { sensors }\end{array}$ & $\begin{array}{c}\text { Average } \\
\text { Packet } \\
\text { Delay } \\
\text { (sec) }\end{array}$ \\
\hline \multirow{2}{*}{\begin{tabular}{|} 
Scenario I \\
Homogenous \\
modulation
\end{tabular}} & $\begin{array}{c}\text { DQPSK } \\
80 \mathrm{kbps}\end{array}$ & $41.97 \%$ & 2638 & 3.87 \\
\hline & $\begin{array}{c}\text { BPSK } \\
\text { 40kpps }\end{array}$ & $60.52 \%$ & 4849 & 9.31 \\
\hline \multirow{5}{*}{\begin{tabular}{|c|} 
Scenario II \\
Heterogeneous \\
modulation
\end{tabular}} & $\mathrm{r}=10(\mathrm{~m})$ & $60.50 \%$ & 4861 & 8.52 \\
\hline & $\mathrm{r}=20(\mathrm{~m})$ & $60.37 \%$ & 4861 & 7.93 \\
\hline & $\mathrm{r}=30(\mathrm{~m})$ & $59.20 \%$ & 4861 & 7.04 \\
\hline & $\mathrm{r}=40(\mathrm{~m})$ & $54.99 \%$ & 4606 & 5.91 \\
\hline & $r=50(m)$ & $44.77 \%$ & 3182 & 4.78 \\
\hline
\end{tabular}

a time frame should be transmitted by the end of that frame. Hence,

$$
\mu_{i} \cdot t_{\text {slot }} \cdot n_{i} \geq \lambda_{i} \cdot t_{\text {slot }} \cdot N_{T} \Rightarrow \frac{N_{T}}{n_{i}} \leq \frac{\mu_{i}}{\lambda_{i}} \quad, i=1,2, \ldots, N
$$

Since $N$ sensors share the same frequency channel,

$$
N_{T}=\sum_{i=1}^{N} n_{i}, \quad n_{i} \geq 1
$$

Given the total bandwidth, selecting the number of frequency channels will set the channel bandwidth and, subsequently, given the type of modulation, will determine the (maximum) bit rate. Hence, according to equation (7), the ratio of the number of slots per frame to the number of slots to be assigned to sensor $i$ has an upper bound. Moreover, both of these parameters, $N_{T}$ and $n_{i}$, depend on the number of sensors sharing a frequency channel through equation (8). Based on constraints (7) and (8) for every sensor $i$, parameters of the slot arrangement, i.e. $N_{T}$ and $n_{i}$, can be obtained.

Now given the slot arrangement, and the temporal variation, shown in Figure 2, and using Little's theorem [14], the average delay for sensor-to-EAR communication for a packet can be obtained as:

$$
\text { delay }_{\text {sensor }(i)-E A R}=\frac{1}{2}\left(1+\frac{\lambda_{i}}{\mu_{i}-\lambda_{i}}\right) \frac{t_{\text {vac }}(i)^{2}}{t_{\text {frame }}}
$$

where $t_{\text {vac }}(i)=\left(N_{T}-n_{j}\right) t_{\text {slot }}$ is the server vacation time, i.e., the time between the end and the start of the two consecutive TDMA channel accesses for sensor $i$.

\subsection{Trade off between lifetime and delay}

As mentioned earlier, there is a trade-off between the modulation power efficiency vs. bandwidth efficiency. Therefore, using the most power efficient or the most bandwidth efficient modulation for all sensors in the network may not be feasible. In general, using less bandwidth efficient modulations like BPSK for increasing the lifetime of the system will force us to use smaller bit rate for data transmission. Therefore, in order to mitigate required sensing traffic, based on Equations (7) and (8), we need to either consider longer TDMA slots or more number of slots per node. This results in larger end-to-end delay.

For the synchronization purposes across the network a common time slot duration should be considered for all wireless links. If the time slot duration is pre-determined based on the less bandwidth efficient modulation with the lower bit rate, there will be channel waist for the nodes with higher bit rate. However, if the time slot duration is decided based on the nodes with higher bit rates, more than one TDMA channel should be assigned to the other nodes with less bandwidth efficient modulation schemes.

\section{Simulation Scenarios and Results}

We consider a hierarchical WSN in an area of $200 \times 200 \mathrm{~m}^{2}$, consisting of 4 clusters, each with 500 sensors distributed randomly using uniform distribution. EARs are placed at the center of each quadrant. The packet size is set to 128 bytes, while the average sensing rate is set to 0.6 packets per sec. The initial battery energy level of each sensor is $2 \mathrm{~kJ}$. Total frequency bandwidth for sensor-to-EAR communication in each cluster is set to $500 \mathrm{KHz}$, which is divided into 10 frequency channels. The path loss exponent is set to 3 . We consider enough time slots, so as to satisfy equation (7) and avoid any cochannel interference. Also to avoid interference from neighboring clusters, different frequency bands are assigned to the different clusters. BPSK and DQPSK modulations are selected. To achieve $0.01 \%$ BER and assuming additive white Gaussian noise channels, the required $E_{b} / N_{0}$ for these modulations are obtained as 8.4 and $11 \mathrm{~dB}$, respectively [12]. To efficiently use the channel bandwidth, the bit rate for sensor-to-EAR links with DQPSK modulation is set to 80 kbps which is smaller than $2 \times 50 \mathrm{KHz}$ (i.e., $2 *$ bandwidth, cf. Table 1 ), and for the BPSK links is set to 40 kbps. The 10 $\mathrm{KHz}$ margin in bandwidth is assumed to accommodate the extra bandwidth required in practice due to presence of inter-symbol interference [12]. Two modulation selection scenarios are considered.

Scenario I (Homogeneous Modulation): Two cases are considered, where DQPSK or BPSK is used in all sensors. Two time slots and one time slot per sensor are allocated for the case of BPSK and DQPSK respectively. Given the bit rates and sensing rate specified above, time slots of size $0.15 \mathrm{sec}$ (100 slots for BPSK and 50 slots for DQPSK), satisfy constraint (7) with the result that all time slots are fully utilized.

Scenario II (Heterogeneous Modulation): DQPSK is used for the centrally located sensors within $r$ meters distance from their assigned EAR and BPSK is assigned to farther away nodes. Different values of $r$ 
are considered. The maximum bit rate for the sensors with BPSK modulation is assumed to be $40 \mathrm{kbps}$, while sensors employing DQPSK transmit at $80 \mathrm{kbps}$. To fully utilize the channels in order to reduce the delay, each time slot is set to $0.15 \mathrm{sec}$ and respectively one slot and two slots per frame are allocated to the sensors with DQPSK and BPSK modulations.

Table 2 provides the results comparing the lifetime and the average delay. For example, the lifetime is improved by about $75 \%$ for heterogeneous modulation with $\mathrm{r}=40 \mathrm{~m}$ versus scenario $\mathrm{I}$ with DQPSK modulation. Also, due to the trade-off between bandwidth efficiency and power efficiency, it can be observed that Scenario II with $\mathrm{r}=40 \mathrm{~m}$ results in about $52 \%$ increase in delay compared to DQPSK case.

Figure 3 shows the delay and lifetime variations for simulation scenario II when the radius $r$ is changed. Note that changing $r$ affects the distribution of sensors for each modulation scheme. Finally, Figure 4 compares the spatial distribution of remaining energy (normalized with respect to initial energy) at the same instance of simulation time for homogeneous and heterogeneous modulation schemes. Results show how heterogeneous modulation scheme can balance the energy dissipation across the network.

\section{Concluding Remarks}

We studied how a location-aware heterogeneous modulation scheme can affect the spatial energy distribution and the overall data delivery delay in a hierarchical wireless sensor network. Specifically, we showed how the modulation selection can increase the lifetime by balancing energy dissipation between the sensors. We studied the inherent trade-off between bandwidth and power efficiency in various modulation schemes and investigated how a slot arrangement scheme can be used to mitigate the effects of less bandwidth efficient modulations on the delay.

\section{References}

[1] Y. Yu, B. Krishnamachari, and V.K. Prasanna, "Energylatency tradeoffs for data gathering in wireless sensor networks," IEEE Infocom, 2004.

[2] Santashil PalChaudhuri, Rajnish Kumar, Richard Baraniuk, David B. Johnson, “Design of adaptive overlays for multi-scale communication in sensor networks, IEEEDCOSS, June 2005.

[3] V. P. Mhatre, C. Rosenberg, D, Kofman, R. Mazumdar, and N. Shroff, "A minimum cost heterogeneous sensor network with lifetime constraint," IEEE Transactions on Mobile Computing, Vol. 4, No1, pp. 4-15, 2005.

[4] A. Iranli, M. Maleki, and M. Pedram, "Energy-efficient strategies for deployment of a two-level wireless sensor network,” SLPED, Aug. 2005, pp. 233-238.

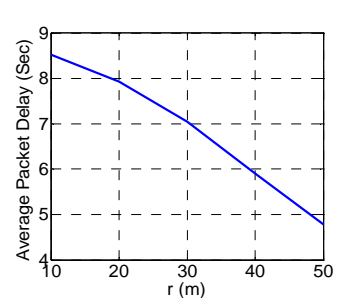

(a)

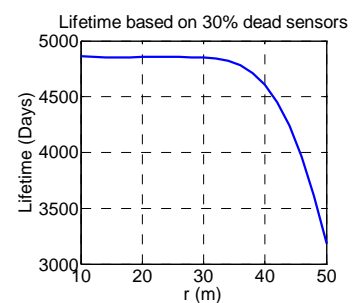

(b)
Figure 3: Scenario II. (a) Average delay variation (b) Lifetime variation.

[5] M. Soltan, M. Maleki, and M. Pedram, "Lifetime-aware hierarchical wireless sensor network architecture with mobile overlays,” IEEE RWS, Jan. 2007, pp. 325-328.

[6] V. Raghunathan, C. Schurgers, S. Park, and M. Srivastava, "Energy-Aware wireless microsensor networks," IEEE Signal Processing Magazine, vol. 19, 2002, pp. 40-50. [7] H. kwon, T. H. Kim, S. Choi, and B. G. Lee, "A crosslayer strategy for energy-efficient reliable delivery in wireless sensor networks," IEEE Transactions on Wireless Communications, vol. 5, no. 12, 2006, pp. 3689-3699.

[8] C. Schurgers, O. Aberborne, and M. Srivastava, "Modulation scaling for energy-aware communication systems,” ISLPED, 2001, pp. 96-99.

[9] Z. Yang, Y. Yuan, J. He, and W. Chen, "Adaptive modulation scaling scheme for wireless sensor networks," IEICE Transactions on Communications, 2005, pp. 882-889.

[10] J. J. E. Garzás, C. B. Calzón, and G Armada, “An Energy-Efficient Adaptive Modulation Suitable for Wireless Sensor Networks with SER and Throughput Constraints," EURASIP Journal on Wireless Communications and Networking, 2007.

[11] J. G Proakis., Digital Communications, McGraw-Hill Inc., Third Edition, 1995.

[12] F. Xiong, Digital Modulation Techniques, Artech House, 2000.

[13] C. E. Shannon, "A mathematical theory of communication," The Bell System Technical Journal, Vol. 27, July-October 1948, pp. 379-423, 623-656.

[14] D. Bertsekas, and R. Gallager, Data Networks, Prentice Hall, Second Edition, 1992.

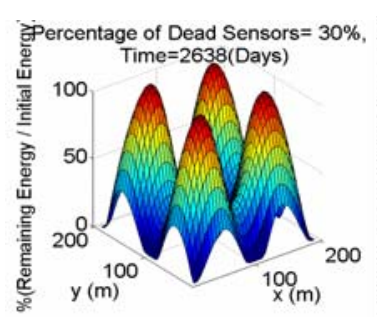

(a)

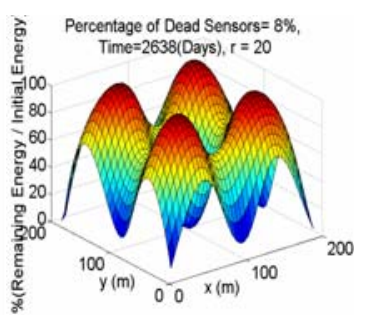

(b)
Figure 4: Spatial distribution of remaining energy over initial energy: (a) Scenario I (DQPSK)(b) Scenario II ( $r=20 \mathrm{~m})$. 